\title{
Supercapacitive performance of vanadium sulfide deposited on stainless steel mesh: effect of etching
}

\author{
Swati J. Patil ${ }^{1}$, Rahul B. Pujari ${ }^{1}$, Tianfeng Hou ${ }^{1}$, Jongsung Park ${ }^{1,2}$ and Dong-Weon Lee ${ }^{1,3^{*}}$ (i)
}

\begin{abstract}
Vanadium sulfide $\left(V_{2}\right)$ nanomaterials have been deposited on surface-modified stainless steel mesh (SMSSM) by facial hydrothermal method and subsequent effects of acidic etching on surface morphology and supercapacitive performance of the electrode were studied. The acid etching process improves the coupling interaction between the stainless steel mesh and active materials. The optimized $1 \%$ etched SMSSM with VS $2\left(1 \% / \mathrm{NS}_{2}\right)$ loaded electrode reveals the porous fine nanoparticles composed surface nanostructure. The prepared $1 \% / \mathrm{NS}_{2}$ electrode exhibited an excellent areal capacitance of $45.83 \mathrm{mF} / \mathrm{cm}^{2}$, which is two times higher than that of a non-etched VS$S_{2}$ loaded electrode. These results showed that the acidic etching process significantly improved the surface nanostructure of the $\mathrm{VS}_{2}$ material that improved the capacitance of the SMSSM substrate.
\end{abstract}

Keywords: Vanadium sulfide $\left(\mathrm{VS}_{2}\right)$, Acidic etching, Areal capacitance

\section{Introduction}

The rapid development of alternative energy storage/conversion systems has become a major consideration due to the growing concern about environmental issues and the reduction of fossil fuels [1]. Electrochemical capacitors, batteries, supercapacitors are commonly available charge storage devices used in different electronics and portable appliances. In which, electrochemical supercapacitors have excellent electrochemical features, and they bridge the gap between chemical batteries and ordinary capacitors. Supercapacitors have a great pledge to the next generation power system due to their superior properties such as high power density, quick charge and discharge rate, excellent irreversibility, and long life cycle [2]. To date, many efforts have been devoted to the latter using binary and ternary transition metal sulfides and they were well-considered and studied [3-5]. Out of which,

\footnotetext{
*Correspondence: mems@jun.ac.kr

${ }^{1}$ MEMS and Nanotechnology Laboratory, Graduate School of Mechanical Engineering, Chonnam National University, Gwangju 500-757, Republic of Korea

Full list of author information is available at the end of the article
}

transition metal dichalcogenides such as $\mathrm{MoS}_{2}, \mathrm{VS}_{2}, \mathrm{WS}_{2}$, $\mathrm{CoS}_{2}$ and $\mathrm{NiS}_{2}$ [6-10] offer two-dimensional (2D) layered structure and it has been considerably investigated for electrochemical application. Strangely, exfoliated monolayer transition metal dichalcogenides manifest several properties from bulk materials and exhibit the high specific surface area, good electrical conductivity, and excellent chemical versatility $[11,12]$.

$\mathrm{VS}_{2}$ is one of the transition metal dichalcogenide electrode materials that has been recently used in electrochemical capacitors due to their layered structure, and diverse chemical and physical properties [13]. In the $\mathrm{VS}_{2}$, $\mathrm{V}$ is sandwiched in between the $\mathrm{S}$ layer and has a large interlayer space of $5.76 \AA$, which makes it a complete stacked entity through the force of Van der Waals [14]. The aforementioned framework provides wide plenty of open electrolyte ion channels with upgrades intercalation/deintercalation process and metallic properties to intensify the charge transfer kinetics. However, the electrochemical performance of the supercapacitor not only depends on the electrode material but also depends upon the current collector, electrolyte and interface 
contact between the electrode/electrolyte. Generally, nanomaterials were deposited on substrate electrodes that have a smooth surface and comparably impenetrable microstructures that restrict their electrochemical performance. The solution has been paid to improve the surface adherence of the electrode using the surface etching process. The surface etching is one of the best ways to improve the surface area, porosity, and wettability of the electrode $[15,16]$. The electrochemical performance of the electrode drastically improved due to the tuning of the electrode surface area that enhances the more electroactive sites for electrochemical reactions.

In the present work, $\mathrm{VS}_{2}$ was hydrothermally prepared on the cost-effective surface-modified stainless steel mesh (SMSSM) substrate. The effects of surface modification with acid etching on the microstructure, and electrochemical properties of the $\mathrm{VS}_{2}$ films were investigated. The electrochemical characteristics were conducted through cyclic voltammetry, charge/discharge and impedance techniques. $\mathrm{VS}_{2}$ deposited on etched SMSSM substrate has the merit of advantages with uniform nanomaterial growth is highly desirable for energy storage applications.

\section{Experimental section}

\section{Surface modification and synthesis of $\mathrm{VS}_{2}$}

The chemical, Ammonium metavanadate $\left(\mathrm{NH}_{4} \mathrm{VO}_{3}\right.$, 99.99\% trace metals basis), thioacetamide $\left(\mathrm{CH}_{3} \mathrm{CSNH}_{2}\right.$, ACS reagent, $\geq 99.0 \%$ ), Lithium chloride powder ( $\mathrm{LiCl}, \geq 99.98 \%$ trace metals basis), Sulfuric acid $\left(\mathrm{H}_{2} \mathrm{SO}_{4}\right.$, ACS reagent, 95.0-98.0\%) were received from Sigma Aldrich and used without any further purification. Initially, SMSSM was washed with distilled water and then used for surface modification. The etching solution was prepared in the ratio $1 \mathrm{M} \mathrm{H}_{2} \mathrm{SO}_{4}: 1 \mathrm{M} \mathrm{LiCl}$ in distilled water for the SMSSM substrate etching. The surface modification was applied with $0 \%, 0.5 \%, 1 \%$ and $1.5 \%$ etching solution at $100{ }^{\circ} \mathrm{C}$ for $1 \mathrm{~h}$. Further, the hydrothermal process was used for the $\mathrm{VS}_{2}$ nanomaterial loading on etched and non-etched SMSSM substrate. For the preparation of $\mathrm{VS}_{2}$, typically $7.5 \mathrm{mmol} \mathrm{CH}_{3} \mathrm{CSNH}_{2}$ were dissolved in $40 \mathrm{ml}$ distilled water and stirred for $10 \mathrm{~min}$ and then added $1 \mathrm{mmol} \mathrm{NH}_{4} \mathrm{VO}_{3}$. The solution was continuously stirred at $40{ }^{\circ} \mathrm{C}$ for $30 \mathrm{~min}$. Further, the final solution was transferred to the $45 \mathrm{ml}$ Teflon-lined stainless steel autoclave along with the piece of a SMSSM and the hydrothermal reaction kept at $180^{\circ} \mathrm{C}$ for $14 \mathrm{~h}$. After natural cooling to room temperature, the blackish colored SMSSM sample was collected and washed several times by distilled water, dried at $60{ }^{\circ} \mathrm{C}$ for $10 \mathrm{~h}$. For the comparative preparation of $\mathrm{VS}_{2}$ on etched SMSSM, the experiments were conducted on different $(0,0.5 \%$, $1 \%$ and $1.5 \%$ ) etched substrate. The optical images of the etched samples with and without $\mathrm{VS}_{2}$ deposition are shown inset of Fig. $5 \mathrm{f}$.

\section{Results and discussion}

In order to visualize the surface morphology and microstructure of the prepared samples, field emission scanning electron microscopy was used and insight their elemental distribution and chemical composition were analyzed using an energy dispersive spectroscopy (EDS). The surface microstructures of the surface-modified $0 \%$, $0.5 \%, 1 \%$ and $1.5 \%$ etched SMSSM substrate is shown in Fig. 1a-d at low magnification $(10 \mathrm{k} \times)$ and the inset shows the highly magnified images $(100 \mathrm{k} \times)$. The effect of surface etching on the microstructure of the SMSSM substrate can be realized in Fig. 1a-d. The smooth,
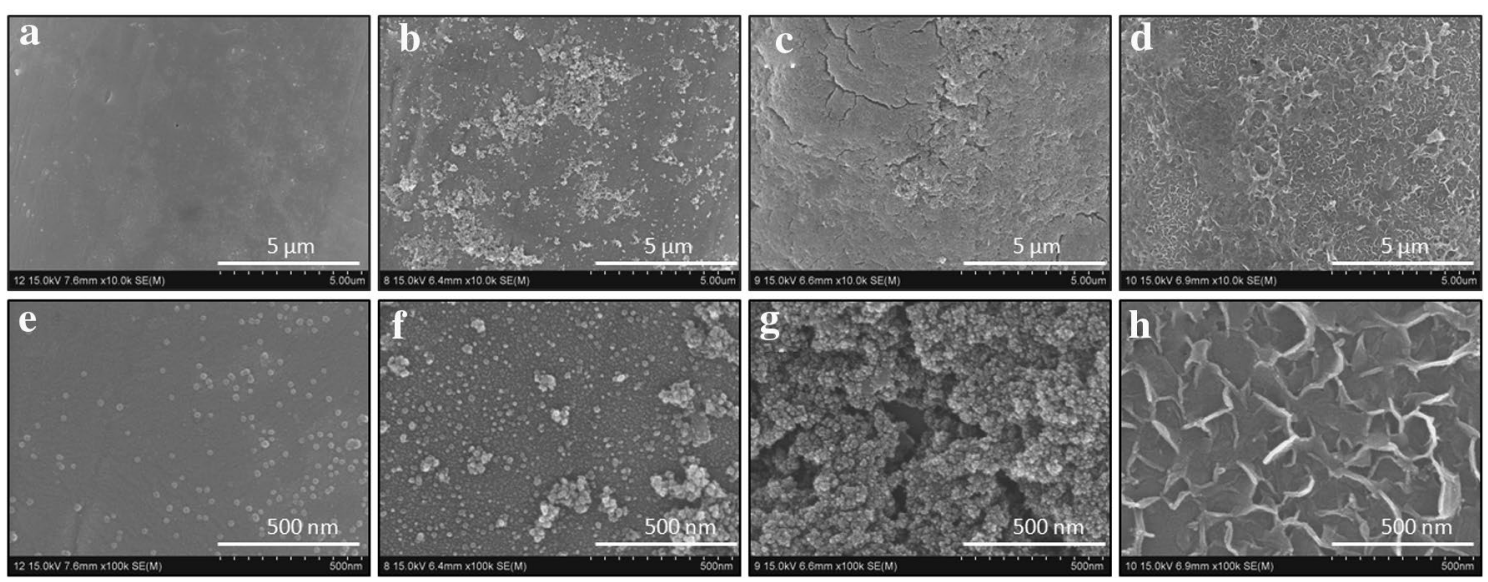

e
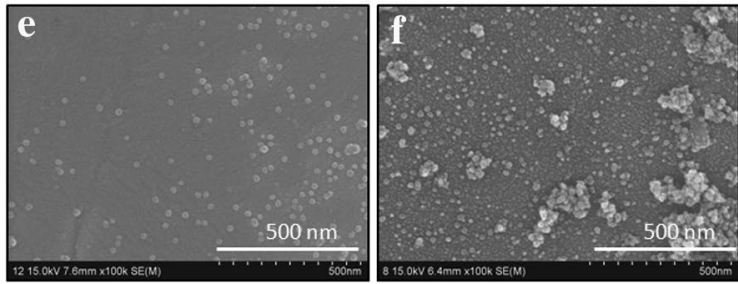

Fig. 1 FESEM images of the surface-modified SMSSM substrate at different etching of a $0 \%, \mathbf{b} 0.5 \%, \mathbf{c} 1 \%$ and $\mathbf{d} 1.5 \%$ at the $\times 10 \mathrm{k}$ magnification (5 $\mathrm{mm}$ scale bar), $\mathbf{e}-\mathbf{h}$ the magnified local images at high magnifications [×100 k magnification ( $\mathrm{nm}$ scale bar)] 
compact surface is observed for bare SMSSM (Fig. 1a). The smooth surface becomes more porous with increasing the surface etching percentage from 0 to $1.5 \%$. As seen in Fig. 1c, the entire surface becomes more porous and exhibits the fine nanospheres like surface structure. Fig. 1e-h shows the magnified image with a uniform coated SMSSM substrate. For 1.5\% etched SMSSM, the agglomerated nanoflakes structure with non-uniformity is observed. With increasing the etching concentrations, the SSM surface exhibits the granular nano-features with large grains. Overall, comparing the nanostructures of the etched SMSSM, at 1\% etched sample exhibits good electrochemical performance (seen in electrochemical studies Fig. 5a, b).

The 1\% etched SMSSM substrate further used for nanomaterial deposition. The SEM images of the $\mathrm{VS}_{2}$ hydrothermally deposition on $1 \%$ non-etched and etched SMSSM substrate as seen in Fig. 2a-c and $d-f$, respectively. The low magnified SEM images of the $\mathrm{VS}_{2}$ deposited on non-etched and 1\% etched samples visualized nanomaterials were well uniformly grown on the SMSSM substrate. High-magnified SEM images (Fig. 2f) of the etched $1 \% / \mathrm{VS}_{2}$ samples have a porous surface. Also, it is seen that nanoparticles are distributed over the SMSSM. While SEM images for the non-etched/VS ${ }_{2}$ samples as shown in Fig. 2a-c exhibits the smooth microstructure. The etched sample has good porosity and uniform converge of the nanomaterials on the SMSSM substrate. The elemental spectra of the $\mathrm{VS}_{2}$ on non-etched and $1 \%$ etched samples represent the peaks of $\mathrm{V}$ and $\mathrm{S}$ of the deposited material are shown in Fig. 2g, h. Besides, other peaks also observed in the EDS spectrums that are related to the SMSSM substrate for both samples. The atomic weight percentages of 1: 2 for $\mathrm{V}: \mathrm{S}$ has confirmed the formation of $\mathrm{VS}_{2}$. To get more information about the morphological and structural features of the $1 \% / \mathrm{VS}_{2}$ samples, the transmission electron microscopic (TEM) analysis carried out and corresponding results are presented in Fig. 3a, b. As seen in Fig. 3a, the formation of nanoparticle like structure is observed and which are consisting of the SEM analysis. The high-resolution TEM (HRTEM) shows the interlayer spacing between two adjacent monolayers is as large as $0.57 \mathrm{~nm}$ corresponding to the (001) plane of the $\mathrm{VS}_{2}$ (Fig. 3b).

$\mathrm{X}$-ray diffractometer (PANalytical, $\mathrm{Cu}-\mathrm{K} \alpha$ radiation, $\lambda=0.15418 \mathrm{~nm}$ ) was used to determine the crystal structure and phase confirmation of the prepared samples. XRD patterns for the SSM, $1 \%$ etched SSM and $1 \% / \mathrm{VS}_{2}$ SSM samples are shown in Fig. 4. As seen in the XRD spectrum, the major peaks observed at $43.5^{\circ}, 50.7^{\circ}$ and
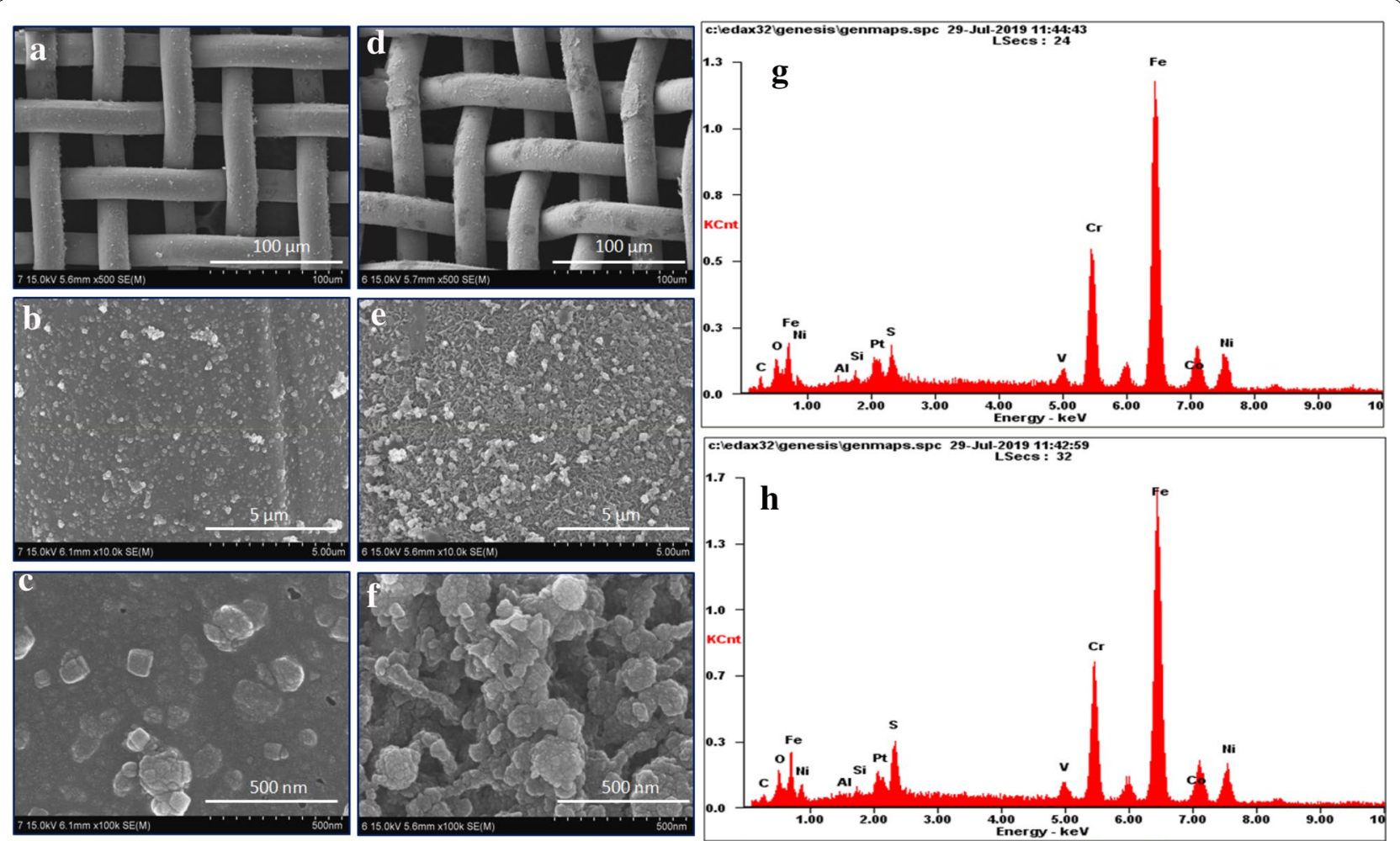

Fig. 2 The low and high-magnified SEM images of VS, on a-c non-etched and $\mathbf{d}-\mathbf{f} 1 \%$ etched SSM substrate. The EDS spectrum of VS 2 on the $\mathbf{g}$ non-etched and $\mathbf{h} 1 \%$ etched SSM substrate 

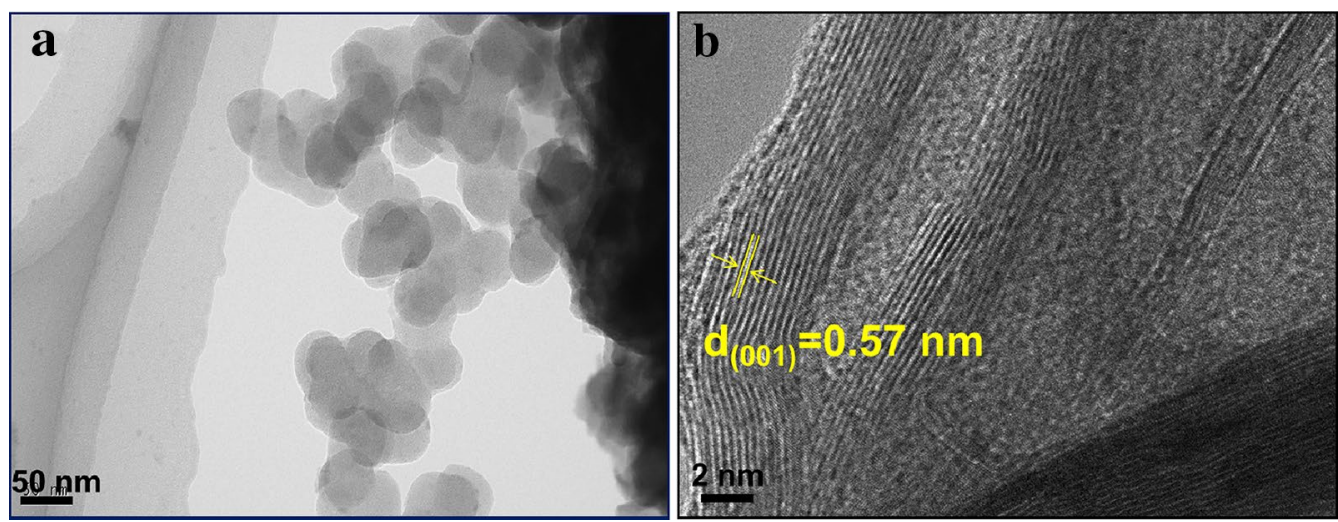

Fig. 3 The low and high-magnified TEM images for $1 \% / \mathrm{NS}_{2}$ sample

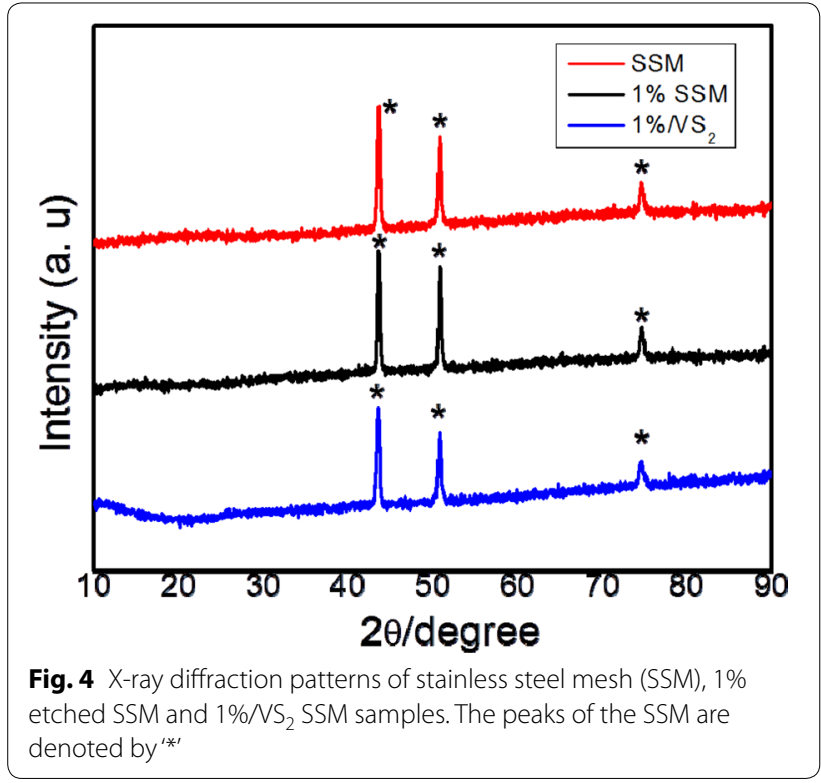

$74.6^{\circ}$ are related to the stainless steel indexing the (111), (200) and (220) lattice planes of austenite phase (JCPDS No. 33-0397) [17]. While, for the $1 \% / \mathrm{VS}_{2} \mathrm{SSM}$, the intensity of the pattern is weakened. Therefore, we can conclude that only the peaks of SMSSM substrate are detected which may be explained by the nanocrystalline of the $1 \% / \mathrm{VS}_{2}$ sample.

\section{Electrochemical studies}

The electrochemical measurements of the prepared samples were conducted in the three-electrode setup in IVIUM technologies (Eindhoven, The Netherlands) designed electrochemical impedance analyzers. SMSSM substrate with supported prepared material with $1 \mathrm{~cm}^{2}$ active area serves as a working electrode, platinum plate serve as a counter electrode. $\mathrm{Ag} / \mathrm{AgCl}$ serves as a reference electrode with $1 \mathrm{M} \mathrm{Na}_{2} \mathrm{SO}_{4}$ as an electrolyte solution. The cyclic voltammetry $(\mathrm{CV})$ and charge/discharge (GCD) tests were investigated with different scan rates. Electrochemical impedance spectroscopy (EIS) was performed in the frequency range of 0.1 to $1 \mathrm{M} \mathrm{Hz}$. The areal capacitance of the prepared materials was calculated using the following equation at constant charge/discharge current density.

$$
C_{A}=\frac{I \times t_{d}}{V \times A}
$$

where, $C_{A}$ is the areal capacitance of the prepared electrode material. $V$ and $I$ are the potential windows and current density. $A$ is the area of active electrode material $\left(\mathrm{cm}^{2}\right)$. The electrochemical studies and effect of etching of the different etched samples was investigated in cyclic voltammetry and impedance analysis. Figure 5a shows the cyclic voltammograms at constant scan rate of $20 \mathrm{mV} / \mathrm{s}$ for the $0 \%, 0.5 \%, 1 \%$ and $1.5 \%$ etched SMSSM samples. It is clearly seen that with etching concentration, the current density of the electrode increased and then decreased. The current density of the $1 \%$ etched sample was increased ten times higher than without the etched sample. In addition, for $1 \%$ etched electrode exhibits the maximum utilized area under the CV curve suggesting may have a good charge storage capacity of the etched sample. The areal capacitance of the surfacemodified samples further calculated from Eq. (1). The calculated corresponding areal capacitances of the etched samples are shown in Fig. 5b.

The etched sample reveals the excellent electrochemical performance for $1 \%$ etching with an areal capacitance of $3.8 \mathrm{mF} / \mathrm{cm}^{2}$. The other samples with $0 \%, 0.5 \%$, and $1.5 \%$ etching, the calculated areal capacitances are found to be $0.13,1.3$, and $2.7 \mathrm{mF} / \mathrm{cm}^{2}$, respectively. Further, 

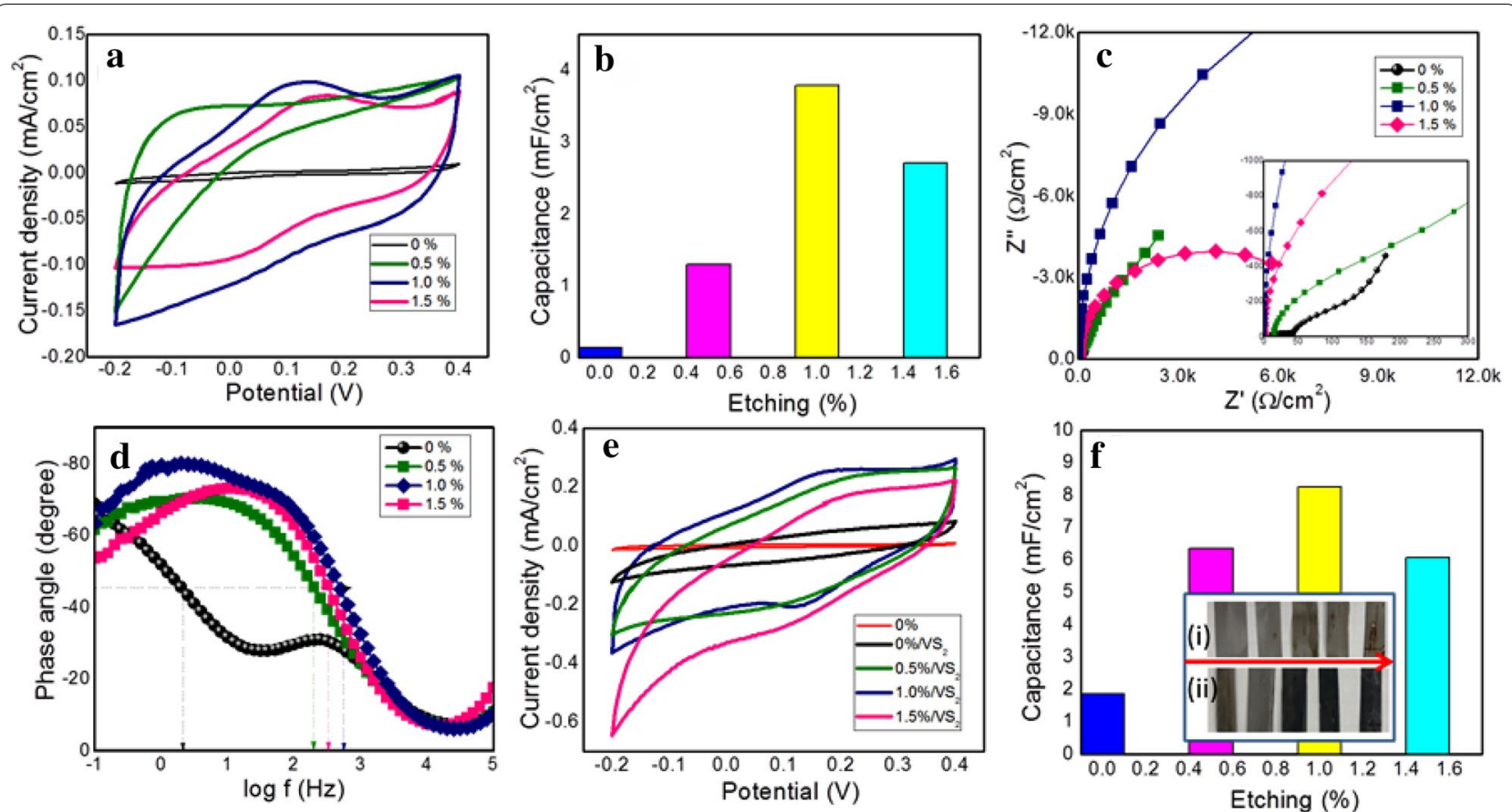

Fig. 5 The electrochemical characterizations of the surface-modified SSM samples; a cyclic voltammograms of the SMSSM substrate at different etching concentrations, $\mathbf{b}$ the graph of areal capacitance as a function of etching concentration. Electrochemical impedance results in the $0.1 \mathrm{~Hz}$ to $100 \mathrm{KHz}$ frequency range; c Nyquist plot for all prepared samples. Inset shows the enlarged view of the Nyquist plot and $\mathbf{d}$ Bode phase angle plots for etched SMSSM substrates. e Cyclic voltammogram at a scan rate of $20 \mathrm{mV} / \mathrm{s}$ scan rate for the VS, nanomaterial deposited on etched SMSSM and corresponding calculated areal capacitances as seen in $\mathbf{f}$. Inset shows the photographs of etched substrates $(0,0.5,1$ and $1.5 \%$ samples); (i) without (ii) with $\mathrm{VS}_{2}$ deposition

the corresponding impedance dataset was conducted to study internal resistance, charge transfer resistance and Warburg impedance of the etched samples. Nyquist plots for the different etched samples are illustrated in Fig. $5 \mathrm{c}$ and its enlarged view as a seen inset of the plot. The non-etched sample reveals the large charge transfer resistance, with increasing the etching concentration the surface becomes more porous and conductivity improved and hence the resistance gradually decreased. The charge transfer resistance diminishes from $40.44 \Omega$ / $\mathrm{cm}^{2}$ for $0 \%$ etched to $1.2 \Omega / \mathrm{cm}^{2}$ for a $1 \%$ etched sample. The smaller charge transfer resistance of $1 \%$ etched sample demonstrates faster electron charge transfer kinetic which is due to the highly porous nature of the sample. The further reaction time constant was calculated from the Bode plot for each etched sample. The Bode phase angle plot for $0,0.5,1$ and $1.5 \%$ etched samples as seen in Fig. 5d. It is seen that, with increasing the etching concentration, the charge transfer resistance of the samples reduces significantly, and the phase angle goes up suddenly indicating the fast reaction rate and a smaller relaxation time constant [18]. The relaxation time constant are found to be $0.48,0.0051,0.0015$ and $0.002 \mathrm{~s}$ for the $0,0.5$, 1 and $1.5 \%$ etched samples, respectively. In addition, the electrochemical measurements were conducted with the $\mathrm{VS}_{2}$ nanomaterial deposited on an individual etched sample. The comparative cyclic voltammograms of the $\mathrm{VS}_{2}$ nanomaterial deposited on etched samples are shown in Fig. 5e. The current density of the electrodes increased with the etching percentage increasing due to the surface electroactive sites opened that effectively enhanced the electrochemical performance of the electrodes. The charge storage capacity of $\mathrm{VS}_{2}$ nanomaterial deposited etched samples were calculated and results are displayed in Fig. 5f. The areal capacitance of 1.7, 6.7, 8.4 and 6.25 $\mathrm{mF} / \mathrm{cm}^{2}$ were obtained for the $0 \% / \mathrm{VS}_{2}, 0.5 \% / \mathrm{VS}_{2}, 1 \% / \mathrm{VS}_{2}$ and $1.5 \% / \mathrm{VS}_{2}$, respectively. Therefore, it is confirmed that a $1 \%$ etched electrode reveals the superior electrochemical performance with good electric conductivity and low charge transfer resistance once compare with less or more etched electrode samples.

Figure 6a illustrates the cyclic voltammograms of the $1 \% / \mathrm{VS}_{2}$ electrode at different scanning rates ranging from 2 to $100 \mathrm{mV} / \mathrm{s}$ in -0.2 to $0.4 \mathrm{~V}$ vs $\mathrm{Ag} / \mathrm{AgCl}$. The rectangular-shaped CV curves with a large area under curve exhibit good charge storage capacity of the $1 \% / \mathrm{VS}_{2}$ electrode. The areal capacitance versus the scan rate of the $1 \% / \mathrm{VS}_{2}$ electrode is shown in Fig. 6 b. The maximum areal 

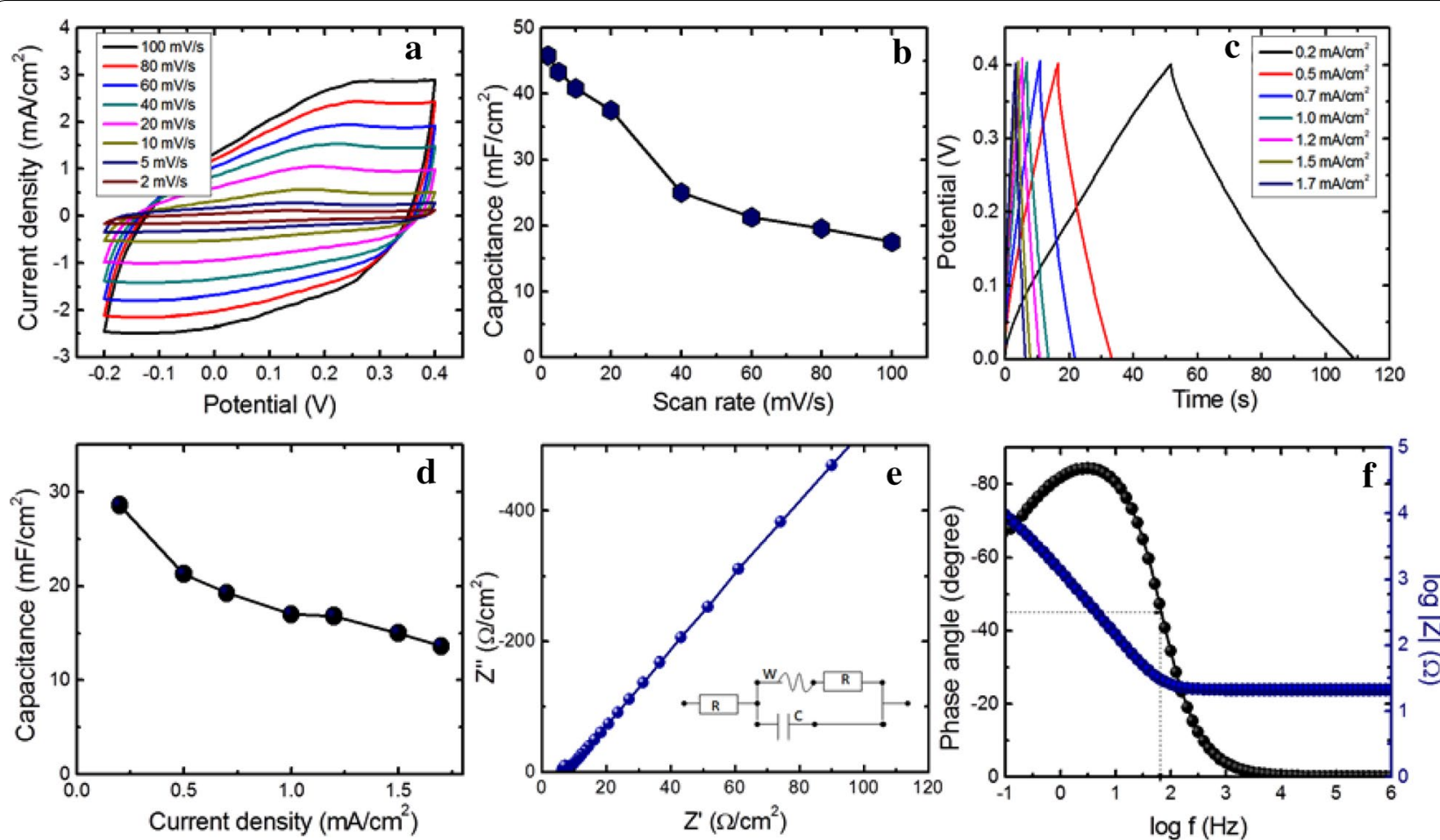

Fig. 6 a Cyclic voltammograms of the $1 \% / \mathrm{NS}_{2}$ electrode recorded at different scan rates ranging from 2 to $100 \mathrm{mV} / \mathrm{s}$ scan rate. $\mathbf{b}$ Areal capacitance of the $1 \% / \mathrm{NS}_{2}$ electrode as a function of scan rates. c The charge/discharge profiles of the $1 \% / \mathrm{NS}_{2}$ electrode at various current densities recorded in the 0 to $0.4 \mathrm{~V}$ vs Ag/AgCl potential window. $\mathbf{d}$ The capacitances of the $1 \% / \mathrm{NS}_{2}$ electrode calculated at a current density ranging from 0.2 to $1.7 \mathrm{~mA} /$ $\mathrm{cm}^{2}$. e The Nyquist plot of the $1 \% / \mathrm{NS}_{2}$ electrode and the inset shows the equivalent circuit used to fit the impedance data. f The Bode phase and magnitude plot for the $1 \% / \mathrm{NS}_{2}$ electrode

capacitance of $45.83 \mathrm{mF} / \mathrm{cm}^{2}$ at $2 \mathrm{mV} / \mathrm{s}$ scan rate and it decreased to $18.5 \mathrm{~F} / \mathrm{cm}^{2}$ at $100 \mathrm{mV} / \mathrm{s}$ scan rate showing the $40.1 \%$ rate capability of the electrode. With an increase in the scan rate, the capacitances of the electrode decreased due to the redox reaction response in the bulk material are limited. While, at a low scan rate, the electroactive material is fully utilized, resulting in high capacitance $[5,19]$. Further, the charge/discharge measurements of the $1 \% / \mathrm{VS}_{2}$ electrode were conducted in different charge/discharge current densities (Fig. 6c). The triangular shape of the charge/discharge curves of the $1 \% / \mathrm{VS}_{2}$ electrode showing the capacitive behavior of the material. The areal capacitances of 28.7, 21.25, 17, 15 and $13.8 \mathrm{mF} / \mathrm{cm}^{2}$ are calculated at current densities of $0.2,0.5,1,1.5$ and $1.7 \mathrm{mF} / \mathrm{cm}^{2}$, respectively with $48.1 \%$ rate capability (Fig. 6d) from the charge/discharge curves. It means that the surface etching can improve the areal capacitance of the electrode, which is nearly two times higher than $\mathrm{VS}_{2}$ ultrathin nanosheets [20].

Electrochemical impedance analyses were investigated to analyze the capacitive properties of the $1 \% /$ $\mathrm{VS}_{2}$ electrode in the three-electrode system in a 0.1 to $1 \mathrm{M} \mathrm{Hz}$ frequency range. The Nyquist plot of the $1 \% / \mathrm{VS}_{2}$ electrode as seen in Fig. 6e consists of three regions; (1) the equivalent series resistance in the highfrequency region is related to the interfacial resistance, electrolyte resistance and intrinsic resistance of the electrode material. (2) In the middle frequency region, the charge transfer resistance, and (3) in the low-frequency region, the straight line represents the Warburg impedance related to the ionic diffusion [21]. The electrolyte resistance of $2.09 \Omega / \mathrm{cm}^{2}$ and charge transfer resistance of $6.1 \Omega / \mathrm{cm}^{2}$ is observed for a $1 \% / \mathrm{VS}_{2}$ electrode. The Bode phase angle and magnitude plot of the $1 \% / \mathrm{VS}_{2}$ electrode is shown in Fig. $6 \mathrm{f}$. The phase angle tends to $-90^{\circ}$ at lower frequency region indicating the capacitive type nature of the electrode material. In addition, the Bode magnitude plot exhibits the log $|\mathrm{Z}|$ as a function of frequency (Fig. 6f). The determined relaxation time $\left(\mathrm{t}=1 / \mathrm{f}_{0}\right)$ constant is $0.015 \mathrm{~s}$ at $63.5 \mathrm{~Hz}$ at mid-high frequency indicating the fast reaction time constant, where the $1 \% / \mathrm{VS}_{2}$ electrode shows resistive to capacitive behavior. Bode magnitude plot reveals that a $1 \% / \mathrm{VS}_{2}$ electrode shows a better capacitive performance in the low-frequency range. 


\section{Conclusions}

In summary, the surface-modified stainless steel mesh substrates have been successfully obtained with different etching concentration and $\mathrm{VS}_{2}$ nanomaterial loaded on the etched substrate. The SEM results proved that the surface porosity and roughness of the samples are improved after etching the SMSSM substrate. In addition, the effect of $\mathrm{VS}_{2}$ nanomaterial loading on nonetched and $1 \%$ etched samples are systematically studied with the help of electrochemical characterizations. The obtained results reveal that the areal capacitance of $1 \% /$ $\mathrm{VS}_{2}$ deposited electrode found to be $17 \mathrm{mF} / \mathrm{cm}^{2}$ that is ten times greater than the non-etched SMSSM electrode.

\section{Abbreviations \\ SMSSM: Surface-modified stainless steel mesh; 2D: Two-dimensional; EDS: Energy dispersive spectroscopy; SEM: Scanning electron microscopy; TEM: Transmission electron microscopy; XRD: X-ray diffraction; CV: Cyclic voltam- metry; GCD: Galvanostatic charge/discharge; CA: Areal capacitance.}

\section{Acknowledgements}

This study was supported by the National Research Foundation of Korea (NRF) grant funded by the Korea government (MSIT) (No. 2017R1E1A1A01074550).

\section{Authors' contributions}

SJP designed the experiment and wrote the overall manuscript. RBP and TH contributed to characterizations and data analyses. D-WL reviewed/edited the manuscript. All authors read and approved the final manuscript.

\section{Funding}

National Research Foundation of Korea (NRF) (No. 2017R1E1A1A01074550).

\section{Availability of data and materials}

All data produced and analyzed in this study are included in this article.

\section{Competing interests}

The authors declare that they have no competing interests.

\section{Author details}

${ }^{1}$ MEMS and Nanotechnology Laboratory, Graduate School of Mechanical Engineering, Chonnam National University, Gwangju 500-757, Republic of Korea. ${ }^{2}$ Department of Precision Mechanical Engineering, Kyungpook National University, Sangju 37224, Korea. ${ }^{3}$ Center for Next-Generation Sensor Research and Development, Chonnam National University, 300 Youngbong, Buk, Gwangju 500-757, Republic of Korea.

Received: 6 November 2019 Accepted: 7 May 2020

Published online: 23 May 2020

\section{References}

1. Giussi Juan M, Lorena Cortez M, Marmisolle Waldemar A, Azzaroni Omar (2019) Practical use of polymer brushes in sustainable energy applications: interfacial nanoarchitectonics for high-efficiency devices. Chem Soc Rev 48:814-849

2. Kouchachvili L, Yaïci W, Entchev W (2018) Hybrid battery/supercapacitor energy storage system for the electric vehicles. J Power Sources 374:237-248
3. $Y u X-Y, Y u L$, David W, Lou X (2016) Metal sulfide hollow nanostructures for electrochemical energy storage. Adv Energy Mater 6:1501333

4. Chen D, Lu M, Li L, Cai D, Li J, Cao J, Han W (2019) Hierarchical coreshell structural $\mathrm{NiMoO}_{4} @ \mathrm{NiS}_{2} / \mathrm{MoS}_{2}$ nanowires fabricated via an in situ sulfurization method for high performance asymmetric supercapacitors. $J$ Mater Chem A 7:21759-21765

5. Patil SJ, Lee D-W (2018) Scalable and ascendant synthesis of carbon cloth coated hierarchical core-shell CoMoS@Co(OH) for flexible and highperformance supercapacitors. J Mater Chem A 6:9592-9603

6. Gopalakrishnan K, Sultan S, Govindaraj A, Rao CNR (2015) Supercapacitors based on composites of PANI with nanosheets of nitrogen-doped RGO, $\mathrm{BC}_{1.5} \mathrm{~N}, \mathrm{MoS}_{2}$ and $\mathrm{WS}_{2}$. Nano Energy 12:52-58

7. Pandit B, Karade S, Sankapal BR (2017) Hexagonal VS, Anchored MWCNTs: first approach to design flexible solid-state symmetric supercapacitor device. ACS Appl Mater Interfaces 9:44880-44891

8. Huang K, Zhang JZ, Shi GW, Liu YM (2014) Hydrothermal synthesis of molybdenum disulfide nanosheets as supercapacitors electrode material. Electrochim Acta 132:397-403

9. Xing JC, Zhu YL, Li MY, Jiao QJ (2014) Hierarchical mesoporous CoS 2 microspheres: morphology-controlled synthesis and their superior pseudocapacitive properties. Electrochim Acta 149:285-292

10. Chen W, Zhang X, Mo LE, Feng Z, Chen S, Zhang X, Zhang Y, Hu L (2019) Ligands induced $\mathrm{NiS}_{2}$ quantum dots for synchronous high specific capacity and robust stability of advanced electrochemical energy storage. Chem Eng J 375:121981

11. Theerthagiri J, Karuppasamy K, Durai G, Rana AUI, Arunachalam P, Sangeetha K, Kuppusami P, Kim H-S (2018) Recent advances in metal chalcogenides ( $M X ; X=S, S e)$ nanostructures for electrochemical supercapacitor applications: a brief review. Nanomaterials 8(4):256

12. Pumera M, Sofer $Z$, Ambrosi A (2014) Layered transition metal dichalcogenides for electrochemical energy generation and storage. J Mater Chem A 2:8981-8987

13. Li L, Li Z, Yoshimura A, Sun C, Wang T, Chen Y et al (2019) Vanadium disulfide flakes with nanolayered titanium disulfide coating as cathode materials in lithium-ion batteries. Nat Commun 10:1764

14. Sun R, Wei Q, Sheng J, Shi C, An Q, Liu S, Mai L (2017) Novel layer-by-layer stacked $\mathrm{VS}_{2}$ nanosheets with intercalation pseudocapacitance for highrate sodium ion charge storage. Nano Energy 35:396-404

15. Gao Z, Wu Z, Zhao S, Zhang T, Wang Q (2019) Enhanced capacitive property of HfN film electrode by plasma etching for supercapacitors. Mater Lett 235:148-152

16. Masarapu C, Wei B (2007) Direct growth of aligned multiwalled carbon nanotubes on treated stainless steel substrates. Langmuir 23:9046-9049

17. Wang N, Ma Z, Yao M, Sun J, Hu W (2019) Waste stainless steel mesh anodized under hydrothermal environment for flexible negative electrode of supercapacitor. J Porous Mater 26:1489-1494

18. Yang L, Zhu X, Xiong S, Wu X, Shan Y, Chu PK (2016) Synergistic $\mathrm{WO}_{3} \cdot 2 \mathrm{H}_{2} \mathrm{O}$ nanoplates/WS 2 hybrid catalysts for high-efficiency hydrogen evolution. ACS Appl Mater Interfaces 8(22):13966-13972

19. Patil SJ, Kim JH, Lee DW (2017) Self-assembled Ni3S2//CoNi2S4 nanoarrays for ultra high-performance supercapacitor. Chem Eng J 322:498-509

20. Feng J, Sun X, Wu C, Peng L, Lin C, Hu S, Yang J, Xie Y (2011) Metallic fewlayered VS, ultrathin nanosheets: high two-dimensional conductivity for in-plane supercapacitors. J Am Chem Soc 133:17832-17838

21. Patil SJ, Kim JH, Lee DW (2017) Graphene-nanosheet wrapped cobalt sulphide as a binder free hybrid electrode for asymmetric solid-state supercapacitor. J Power Sources 342:652-665

\section{Publisher's Note}

Springer Nature remains neutral with regard to jurisdictional claims in published maps and institutional affiliations. 